\title{
Sentinel Headache: A Warning Sign Preceding Every Fourth Aneurysmal Subarachnoid Hemorrhage
}

W ith great interest, we read the recent retrospective study of Oda et $\mathrm{al}^{1}$ in the American Journal of Neuroradiology reporting a $33.9 \%$ incidence of minor leaks preceding aneurysmal subarachnoid hemorrhage (aSAH) confirmed by neuroradiologic methods, in which the authors found a much lower incidence of sentinel headache $(\mathrm{SH})$ of $11 \%$ by patient interview. Our data from an ongoing Swiss prospective observational study (ClinicalTrials.gov identifier: NCT02129010) confirms that SH preceding aSAH is not by any means a rare phenomenon. In 8 (24.2\%) of 33 patients, $\mathrm{SH}$ was confirmed by either the patient or next of kin. Contrary to those of Oda et $\mathrm{al}^{1}{ }^{1}$ the data from our study (Table) do not indicate any difference in radiologic bleeding characteristics, such as the presence of intracerebral hemorrhage and a higher rebleeding rate, or worse outcome in patients with $\mathrm{SH}$. Nonetheless, mortality in our SH group was considerably high $(37.5 \%)$, which once again renders distinguishing dangerous headache from innocuous headache on clinical grounds of paramount importance. In the absence of nuchal rigidity, a diminished level of consciousness, or focal neurologic deficits, any sudden onset of atypical headache should raise the suspicion of $\mathrm{SH}$ and should be followed by further investigations. It is therefore important to appreciate the sensitivity and specificity and the lim-

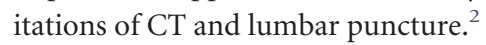

Education on a primary care level is key to correctly identifying $\mathrm{SH}$ and preventing major aSAH with its subsequent morbidity and mortality. For example, a Swedish educational program for local physicians proved to be effective in reducing diagnostic errors by $77 \% .{ }^{3}$ Whether any of our 8 patients confirmed positive for $\mathrm{SH}$ previously sought medical attention was not explicitly investigated by the study protocol. To consolidate awareness among primary care physicians, this short report of prospectively collected data complementing the retrospective study of Oda et $\mathrm{al}^{1}$ is

http://dx.doi.org/10.3174/ajnr.A4467 meant to highlight the importance of $\mathrm{SH}$ preceding aSAH: About every fourth patient with aSAH has preceding symptoms.

\section{ACKNOWLEDGMENTS}

CSF protein INNOTEST ELISA test kits were provided by Innogenetics N.V. (Ghent, Belgium); this funding source has no role in the acquisition of the data stated in the current report.

The current study was approved by the ethics committee, St. Gallen, Switzerland (EKSG 13/011/1B). All study participants or substitute decision makers gave informed consent before taking part. Study protocol can be found at ClinicalTrials.gov (Identifier: NCT02129010).

\section{REFERENCES}

1. Oda S, Shimoda M, Hirayama A, et al. Neuroradiologic diagnosis of minor leak prior to major SAH: diagnosis by T1-FLAIR mismatch. AJNR Am J Neuroradiol 2015 May 14. [Epub ahead of print] CrossRef Medline

2. Edlow JA, Caplan LR. Avoiding pitfalls in the diagnosis of subarachnoid hemorrhage. N Engl J Med 2000;342:29-36 CrossRef Medline

3. Fridriksson S, Hillman J, Landtblom AM, et al. Education of referring doctors about sudden onset headache in subarachnoid hemorrhage: a prospective study. Acta Neurol Scand 2001;103: 238-42 CrossRef Medline 
Characteristics of patients with aneurysmal subarachnoid hemorrhage with and without sentinel headache ${ }^{\mathrm{a}}$

\begin{tabular}{|c|c|c|c|c|c|}
\hline \multirow[b]{2}{*}{ Age (yr) } & \multicolumn{2}{|c|}{ Sentinel Headache } & \multicolumn{2}{|c|}{ No Sentinel Headache } & \multirow{2}{*}{$\frac{P \text { Value }}{.8336^{\mathrm{b}}}$} \\
\hline & 54.1 & $45.2-71.8$ & 53.6 & $49.2-60.8$ & \\
\hline \multicolumn{6}{|l|}{ Admission scores } \\
\hline Glasgow Coma Scale & 13.0 & $7.8-14.4$ & 14.0 & $9.9-13.6$ & $.1912^{\mathrm{b}}$ \\
\hline Hunt and Hess Grade & 3.0 & $2.4-4.1$ & 3.0 & $2.5-3.5$ & $.4507^{\mathrm{b}}$ \\
\hline WFNS grade & 3.0 & $2.2-4.1$ & 2.0 & $2.0-3.2$ & $.2618^{b}$ \\
\hline \multicolumn{6}{|l|}{ Radiologic features } \\
\hline Fisher score & 3.0 & $2.8-3.4$ & 3.0 & $2.9-3.0$ & $.1155^{\mathrm{b}}$ \\
\hline Intraventricular hemorrhage & 5 & $62.5 \%$ & 13 & $52.0 \%$ & $.6992^{c}$ \\
\hline Intracerebral hemorrhage & 2 & $25.0 \%$ & 4 & $16.0 \%$ & $.6162^{c}$ \\
\hline Acute hydrocephalus & 5 & $62.5 \%$ & 19 & $76.0 \%$ & $.6510^{c}$ \\
\hline \multicolumn{6}{|l|}{ Aneurysm location } \\
\hline ACA/AcomA & 2 & $25.0 \%$ & 11 & $44.0 \%$ & $.5436^{\mathrm{d}}$ \\
\hline ICA/PcomA & 3 & $37.5 \%$ & 5 & $20.0 \%$ & \\
\hline MCA & 2 & $25.0 \%$ & 8 & $32.0 \%$ & \\
\hline Posterior circulation & 1 & $12.5 \%$ & 1 & $4.0 \%$ & \\
\hline \multicolumn{6}{|l|}{ Aneurysm characteristics } \\
\hline Dome size (mm) & 6.5 & $4.6-10.6$ & 7.0 & $6.5-7.9$ & $.8155^{\mathrm{b}}$ \\
\hline Neck size (mm) & 3.0 & $1.5-5.5$ & 3.4 & $2.7-5.0$ & $.6447^{\mathrm{b}}$ \\
\hline \multicolumn{6}{|l|}{ Aneurysm occlusion } \\
\hline Surgical occlusion & 4 & $50.0 \%$ & 9 & $36.0 \%$ & $.6573^{d}$ \\
\hline Endovascular occlusion & 3 & $37.5 \%$ & 14 & $56.0 \%$ & \\
\hline None & 1 & $12.5 \%$ & 2 & $8.0 \%$ & \\
\hline \multicolumn{6}{|l|}{ Further treatment } \\
\hline $\mathrm{DHC}$ & 1 & $12.5 \%$ & 2 & $8.0 \%$ & $1.0000^{c}$ \\
\hline VP shunt & 3 & $37.5 \%$ & 7 & $28.0 \%$ & $.6728^{c}$ \\
\hline Balloon dilation & - & $0 \%$ & 8 & $32.0 \%$ & $.1516^{c}$ \\
\hline \multicolumn{6}{|l|}{ Complications } \\
\hline Angiographic vasospasm & 1 & $12.5 \%$ & 16 & $64.0 \%$ & $.0167^{c}$ \\
\hline DIND & - & $0 \%$ & 10 & $40.0 \%$ & $.0715^{c}$ \\
\hline $\mathrm{DCl}$ & - & $0 \%$ & 5 & $20.0 \%$ & $.3023^{c}$ \\
\hline Rebleeding & - & $0 \%$ & 2 & $8.0 \%$ & $1.0000^{\mathrm{C}}$ \\
\hline \multicolumn{6}{|l|}{ Outcome at discharge } \\
\hline Death & 3 & $37.5 \%$ & 5 & $20.0 \%$ & $.3659^{c}$ \\
\hline Unfavorable (mRS 4-5) & - & $0 \%$ & 1 & $4.0 \%$ & $1.0000^{c}$ \\
\hline Favorable (mRS 0-3) & 5 & $62.5 \%$ & 19 & $76.0 \%$ & $.6510^{c}$ \\
\hline $\mathrm{MoCA}$ & 15.0 & $0.4-34.2$ & 18.0 & $11.0-20.9$ & $.9108^{b}$ \\
\hline Total & 8 & $100 \%$ & 25 & $100 \%$ & \\
\hline
\end{tabular}

Note:-ACA indicates anterior cerebral artery; AcomA, anterior communicating artery; DCI, delayed cerebral ischemia; DHC, decompressive hemicraniectomy; DIND, delayed ischemic neurologic deficit; MoCA, Montreal Cognitive Assessment; PcomA, posterior communicating artery; VP, ventriculoperitoneal; WFNS, World Federation of Neurosurgical Societies grading scale.

a The rates of vasospasm and DIND are lower in patients with sentinel headache probably due to higher early mortality in this group. Results are presented as medians with $95 \%$ confidence intervals for nominal variables and in absolute numbers and percentages for categoric variables.

${ }^{b}$ Mann-Whitney test.

${ }^{\mathrm{c}}$ Fisher exact test.

${ }^{\mathrm{d}} \chi^{2}$ test. 